\title{
Modifiable risk factors of acute kidney injury after liver transplantation: a systematic review and meta-analysis
}

\author{
Jian Zhou', Xueying Zhang², Lin Lyu', Xiaojun Ma', Guishen Miao ${ }^{1}$ and Haichen Chu*
}

\begin{abstract}
Background: Acute kidney injury (AKI) is a common and critical complication of liver transplantation (LT), which is associated with increased morbidity, mortality and health care cost. We aimed to identify modifiable risk factors of AKI after LT.
\end{abstract}

Methods: A literature search of Pubmed, EMBASE and Cochrane Databases was performed to identify studies investigating risk factors of AKI after LT. The Newcastle-Ottawa Scale was used to rate study quality. Effect size and 95\% confidence interval were pooled using a random-effect model with inverse-variance method.

Results: Sixty-seven articles with 28,844 patients were included in the meta-analysis. Seventeen modifiable risk factors were found, including overweight, preoperative use of diuretic, preoperative anemia, donation after cardiac death organ, donor $\mathrm{BMI} \geq 30 \mathrm{~kg} / \mathrm{m}^{2}$, ABO-incompatible $\mathrm{LT}$, low graft to recipient body weight ratio, intraoperative hypotension, major bleeding, intraoperative use of vasopressor, large RBC transfusion, postreperfusion syndrome, postoperative use of vasopressors, overexposure to calcineurin inhibitor, calcineurin inhibitor without mycophenolate mofetil, graft dysfunction and infection. A total of 38 articles were included in the systematic review, in which 8 modifiable risk factors and 1 protective factor were additionally associated in single studies with the incidence of AKI after LT.

Conclusions: Effective interventions based on identified modifiable risk factors in the perioperative management and graft allocation and preservation may be promising to reduce the incidence of AKI after LT.

Trial registration: The protocol for this systematic review is registered with PROSPERO (No. CRD42020166918).

Keywords: Acute kidney injury, Liver transplantation, Meta-analysis, Modifiable risk factors

\section{Background}

Acute kidney injury (AKI) is rapid functional or structural kidney abnormality characterized by increased serum creatinine $(\mathrm{Scr})$ or decreased urine volume [1]. The definition of AKI has evolved rapidly from Risk, Injury, Failure, Loss of kidney function, End-stage renal

\footnotetext{
* Correspondence: chiefchu@126.com

'Department of Anesthesiology, The Affiliated Hospital of Qingdao University, School of Clinical Medicine, Qingdao University, No. 59, Haier Road, Qingdao 266100, Shandong Province, China

Full list of author information is available at the end of the article
}

failure (RIFLE) criteria, Acute Kidney Injury Network (AKIN) criteria into Kidney Disease: Improving Global Outcomes (KDIGO) criteria during the past two decades. The KDIGO criteria merges RIFLE criteria and AKIN criteria, encompasses both a relative and an absolute change of Scr and allows a short and an extended time frame for diagnosis [2]. Providing simple and practical definition of AKI, KIDGO criteria gradually became standard criteria, allowing for more consistent estimates of epidemiology. A meta-analysis demonstrated that the incidence and mortality rate of AKI was 21.6 and $23.9 \%$,

C C The Author(s). 2021 Open Access This article is licensed under a Creative Commons Attribution 4.0 International License, which permits use, sharing, adaptation, distribution and reproduction in any medium or format, as long as you give appropriate credit to the original author(s) and the source, provide a link to the Creative Commons licence, and indicate if changes were made. The images or other third party material in this article are included in the article's Creative Commons licence, unless indicated otherwise in a credit line to the material. If material is not included in the article's Creative Commons licence and your intended use is not permitted by statutory regulation or exceeds the permitted use, you will need to obtain permission directly from the copyright holder. To view a copy of this licence, visit http://creativecommons.org/licenses/by/4.0/ The Creative Commons Public Domain Dedication waiver (http://creativecommons.org/publicdomain/zero/1.0/) applies to the data made available in this article, unless otherwise stated in a credit line to the data. 
respectively [3]. These numbers are even higher for patients undergoing liver transplantation (LT), where the incidence of AKI and severe AKI requiring renal replacement therapy (RRT) after LT is up to 40.8 and $7.0 \%$, respectively [4].

AKI is a common and critical complication of LT, which remains particularly prominent among different postoperative organ injuries $[5,6]$. Evidence has indicated that even transient or subclinical AKI is known to be of substantial clinical significance [7]. Previous studies have reported that AKI after LT is not only associated with immediate complications including volume overload, metabolic acidosis, and electrolyte disturbances, but also an increased rate of inferior long-term outcomes such as mortality, graft loss, infection, chronic kidney disease (CKD), prolonged stay in the intensive care unit (ICU), and augmented hospital costs [5-7]. Although much effort has been taken to the treatment of AKI, it does not seem to reverse the natural course of AKI syndrome and effectively improve prognosis [8]. AKI is increasingly recognized as a disease process with continuum of kidney injury instead of a single-hit or freestanding condition [1]. With in-depth research, the past decades have witnessed the shift of attention from treatment to prediction and early detection to avoid repetitive hits and additional damage. Thus, investigating the modifiable risk factors of AKI after LT is of vital importance.

In the past few years, a number of risk factors and predictors of AKI after LT have been reported. However, some of the conclusions remain conflicting and the role of modifiable factors are understudied with insufficient supportive evidence. So far, no comprehensive metaanalysis regarding the modifiable risk factors of AKI after LT has been conducted. Therefore, we performed a systematic review and meta-analysis via an extensive search of observational studies to identify the modifiable risk factors of AKI after LT.

\section{Methods}

This systematic review and meta-analysis was conducted according to the Preferred Reporting Items for Systematic Reviews and Meta-Analysis (PRISMA) statement (see Supplementary 1, Additional File:PRISMA 2009 Checklist) and Meta-analysis Of Observational Studies in Epidemiology (MOOSE) $[9,10]$. The protocol for this systemic review is registered with the international prospective register of systemic reviews (PROSPERO) (No. CRD42020166918).

\section{Study identification and search strategy}

A literature search of Pubmed, EMBASE and Cochrane was performed to identify articles reporting the risk factors of AKI in patients undergoing LT. The research strategy consisted of the key search terms 'liver transplantation' and 'acute kidney injury' and their synonyms, as well as related Mesh terms combined by Boolean operators. The full search strategies for all databases are available in Supplementary 2, Additional File. Only studies published in English were included. In addition, a manual search for conceivably related studies using references of the included articles was also performed.

\section{Inclusion and exclusion criteria}

The inclusion criteria were as follows: (a) observational studies including cohort, case-control and crosssectional studies; (b) studies investigating patients undergoing LT; (c) a minimum of 1 risk factor identified as being associated with AKI after LT, studies illustrating the risk factors of RRT due to AKI after LT were also included as RRT per se is one of the diagnostic criteria of AKI; (d) studies reporting odds ratio (OR) with corresponding 95\% confidence interval (CI) data or enough data to calculate these figures; (e) online full-text available publication. Studies were excluded for the following reasons: (a) studies did not include human subjects; (b) nonoriginal studies (conference abstracts, editorials, letters, reviews, meta-analysis, commentaries or case reports) and duplicated studies; (c) sample size was less than 50; (d) studies exploring new biomarkers or predictors of AKI after LT that are not clinically used. If more than one article were found to have used the same data, we chose the one with higher quality score, and where the quality score was equal, we chose the study with the larger sample size. Retrieved citations were first screened for relevance at the title and/or abstract level, studies remaining after the initial screening were appraised in the full text with respect to the aforementioned inclusion and exclusion criteria. Two authors (XYZ and LL) independently evaluated the eligibility of all studies. If there was disagreement regarding whether to include some articles, these articles would be further evaluated by a third author (JZ) and discussed in detail until an agreement was reached.

\section{Data extraction and quality assessment}

For each article the following data were extracted when available: name of the first author, year of publication, country, cohort source, types of study design, sample size, donor type, surgical technique, baseline patient characteristics, definition and diagnostic criteria of AKI, duration of evaluation, incidence of AKI after LT, risk factor(s) studied, adjustment variables, the statistical methods used for multivariate analysis, effect size and 95\% CI. When both of the univariate OR and the multivariate OR were reported in one study, only multivariate OR were extracted. If the OR was not reported, it was calculated from the original data if possible. When more 
than one definition of AKI were adopted to stratify study population in one study, we only extracted data with the latest diagnostic criteria. Modifiable risk factors refer to risk factors that can be modified by medical interventions or by individual behavior. Clinical variables were also included if they can be modified by medical interventions during perioperative period. Extracted data of the included studies were registered on dedicated electronic forms. The forms were piloted over the first 5 included studies for consistency and discrimination.

The quality of the included studies was assessed by the Newcastle-Ottawa Scale (NOS) [11]. The studies were judged on three broad perspectives: the selection of study populations, the comparability of the populations, and ascertainment of exposure and the outcomes of interest for case-control or cohort studies, respectively. A maximum score of 9 reflects the highest quality. No study was excluded because of a low-quality score. Two authors (XYZ and XJM) performed the data extraction and quality assessment independently. Disagreements were settled by discussion involving a third author (HCC) and consensus was reached on all items finally.

\section{Statistical analysis}

If a risk factor was reported by at least 2 studies in a consistent manner, we would conducted a metaanalysis. Effect size and 95\% CI were pooled using a random-effect model with inverse-variance method $[12,13] . \mathrm{I}^{2}$ statistic and Cochran's Q test were applied to determine the between-study heterogeneity. A value of $\mathrm{I}^{2}$ of $0-25 \%$ represents insignificant heterogeneity, $26-50 \%$ low heterogeneity, $51-75 \%$ moderate heterogeneity and $76-100 \%$ high heterogeneity [14]. In addition to the value of $\mathrm{I}^{2}$, we will also consider strength of evidence for the heterogeneity (CI, chisquared test and/or $P$ value) and the size and direction of effect in the analysis [15]. $P$-values on the Egger's test greater than 0.05 and symmetry of the funnel plot determined the absence of publication bias $(N \geq 10)$ [16]. If significant publication bias was noted, Duval and Tweedie's trim and fill method was used to acquire adjusted values [17]. To minimize heterogeneity, subgroup analyses by diagnostic criteria of AKI, duration of evaluation and statistical method were conducted. Meta-regression analyses $(N \geq 10)$ were also used to assess the potentially important covariates that might exert a substantial impact on between-study heterogeneity. Sensitivity analyses were performed after excluding 1 study at a time to assess the stability of the results and explore the source of heterogeneity. $P<0.05$ was considered statistically significant except where otherwise stated. If data were not available for the meta-analysis or only 1 single study was identified for a given risk factor, these studies were only listed in this systematic review. Statistical analyses were performed using Stata Version 14.0 (StataCorp, Texas, USA).

\section{Results}

\section{Literature search and study selection}

A total of 3273 citations were retrieved after searching PubMed, EMBASE and CENTRAL database. There were 170 full-text articles assessed for eligibility after screening titles and abstracts. After hand-searching the references of included articles and existing reviews and meta-analyses, 1 reference was added. Three articles were excluded due to duplicate data (see Supplemen$\operatorname{tary} 3$, Additional File). In total, 67 articles were eligible for inclusion in the systematic review and meta-analysis (see Supplementary 4, Additional File). Full details of the selection process were presented in Fig. 1.

Characteristics of included studies and quality assessment A total of 67 observational studies published between 2001 and 2019 with 28,844 patients were included. The incidence of AKI after LT ranged from 3.97\% [18] to 71.9\% [19]. Most of the included studies adopted multivariate logistic regression analysis to adjust confounding factors followed by propensity score matching method. The outcome indexes consisted of AKI, and RRT due to AKI. The RIFLE, AKIN, or KDIGO criteria were often used in combination with other scales to assess and classify AKI. The duration of evaluation varied from $12 \mathrm{~h}$ after reperfusion to 3 months after LT. Based on the NOS, the mean quality score of all included studies was 6.686 (standard deviation $=0.633)($ see Supplementary 5, Additional File).

\section{Results of meta-analysis \\ Incidence of AKI after LT}

Overall, the pooled estimated incidence rate of AKI after LT was $37.5 \%$ (95\% CI: $32.3-42.7 \%, \mathrm{I}^{2}=99.5 \%$, Fig. 2 ). Besides, we further did a subgroup analysis based on different diagnostic criteria. The outcomes indicated that prevalence of AKI after LT was 33.5\% (RIFLE), 40.0\% (AKIN), 44.2\% (KDIGO), and 35.2\% (Others), respectively. Meta-regression showed that the publication year did not significantly affect the incidence rate of AKI after LT $(P=0.489$, Fig. 3$)$.

\section{Modifiable risk factors of AKI after LT}

We pooled a forest plot for each factor that was described in at least 2 articles (see Table 1; Details are shown in Supplementary 6, Additional File). Considering the smaller pooled population $(<500)$ reported for some modifiable factors (cadaveric donor liver graft, intraoperative colloidal use, large postoperative red blood cell $[\mathrm{RBC}]$ transfusion, postoperative hypotension), here we 


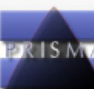

PRISMA 2009 Flow Diagram
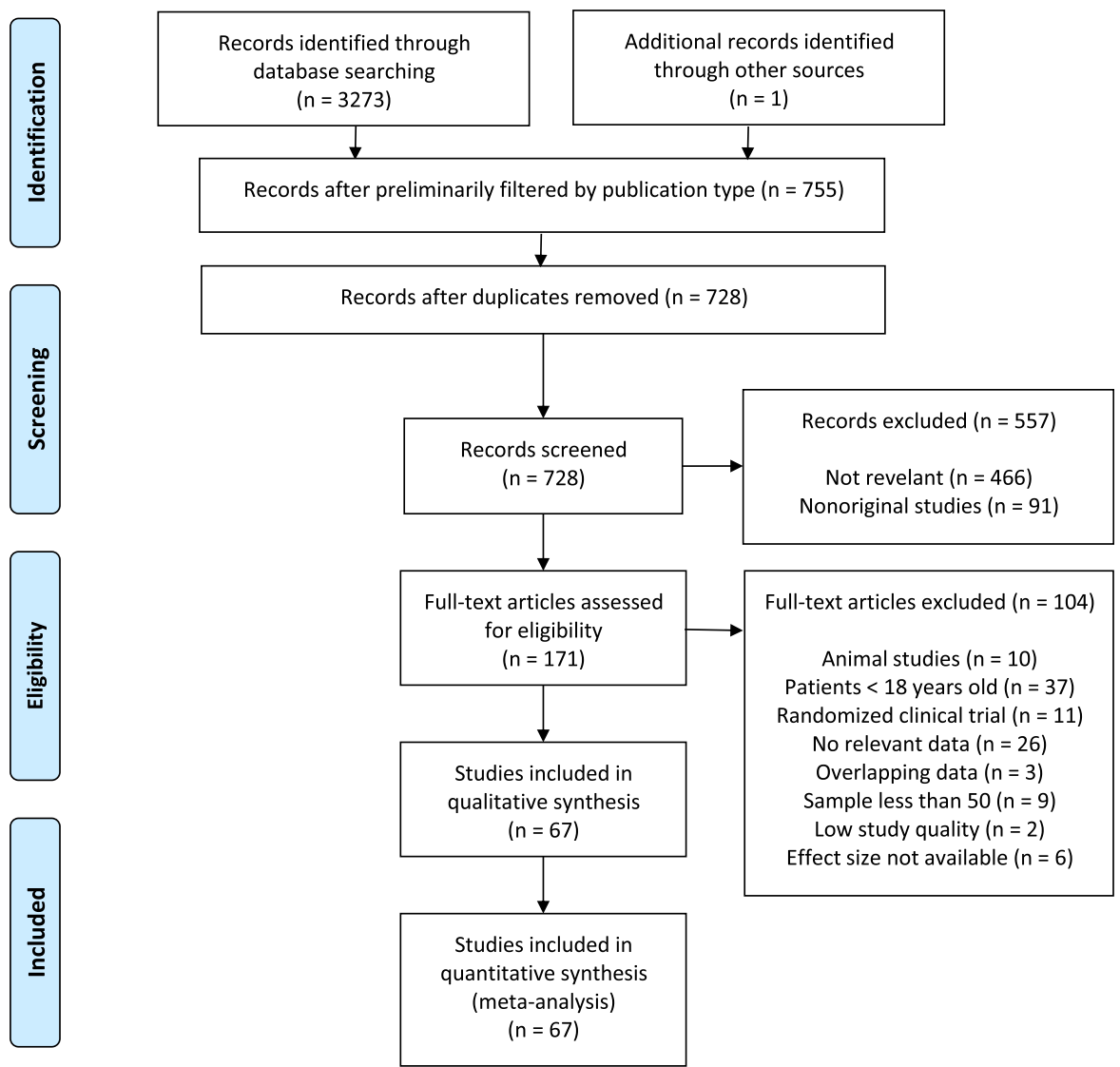

Fig. 1 Flow diagram

only presented the modifiable factors with a relatively large population $(>500)$ to lower the error of estimates. Modifiable factors showing significant associations with AKI after LT are presented in Fig. 4. All of these factors were classified into the following 4 groups: recipient factors, donor and graft factors, surgical factors, postoperative factors.

\section{Recipient factors}

Overweight $\left(\mathrm{OR}=2.437,95 \% \mathrm{CI}=1.629-3.646, \mathrm{I}^{2}=\%\right.$, $P=0.000)$, preoperative use of diuretic $(\mathrm{OR}=2.733,95 \%$ $\left.\mathrm{CI}=1.302-5.739, \quad \mathrm{I}^{2}=41.4 \%, \quad P=0.008\right), \quad$ preoperative anemia $\left(\mathrm{OR}=1.621,95 \% \mathrm{CI}=1.073-2.449, \mathrm{I}^{2}=24.5 \%\right.$, $P=0.022)$ were identified as modifiable risk factors of AKI after LT. Preoperative hypertension and preoperative hypoalbuminemia were not correlated with AKI after LT.

\section{Donor and graft factors}

Donation after cardiac death $(\mathrm{DCD})$ organ $(\mathrm{OR}=2.704$, $\left.95 \% \mathrm{CI}=1.938-3.772, \mathrm{I}^{2}=0.0 \%, P=0.000\right)$, donor body mass index $(\mathrm{BMI}) \geq 30 \mathrm{~kg} / \mathrm{m}^{2} \quad(\mathrm{OR}=2.672, \quad 95 \% \quad \mathrm{CI}=$ 1.173-6.085, $\left.\mathrm{I}^{2}=57.8 \%, P=0.019\right)$, ABO-incompatible LT $\left(\mathrm{OR}=2.761,95 \% \mathrm{CI}=1.602-4.759, \mathrm{I}^{2}=0.0 \%, P=0.000\right)$, low graft weight to recipient body weight ratio $(\mathrm{GW} /$ RBW) $\left(\mathrm{OR}=1.902,95 \% \mathrm{CI}=1.013-3.568, \mathrm{I}^{2}=52.3 \%, P=\right.$ 0.045 ) increased the risk of AKI after LT. However, no significant associations were found for long cold ischemia time (CIT) and long warm ischemia time (WIT).

\section{Surgical factors}

Intraoperative hypotension $(\mathrm{OR}=5.582,95 \% \mathrm{CI}=3.934-$ $\left.7.920, \mathrm{I}^{2}=0.0 \%, P=0.000\right)$, major bleeding $(\mathrm{OR}=2.900$, $\left.95 \% \mathrm{CI}=1.495-5.627, \mathrm{I}^{2}=83.1 \%, P=0.002\right)$, intraoperative use of vasopressor $(\mathrm{OR}=2.079,95 \% \mathrm{CI}=1.492$ $\left.2.899, \mathrm{I}^{2}=70.3 \%, \mathrm{P}=0.000\right)$, large intraoperative $\mathrm{RBC}$ transfusion $\left(\mathrm{OR}=3.124,95 \% \mathrm{CI}=1.986-4.914, \mathrm{I}^{2}=\right.$ $72.8 \%, P=0.000)$, postreperfusion syndrome $(\mathrm{OR}=$ $\left.1.689,95 \% \mathrm{CI}=1.275-2.236, \mathrm{I}^{2}=52.8 \%, P=0.000\right)$ were associated with an increased risk for AKI after LT. Nevertheless, no obvious associations were detected with 


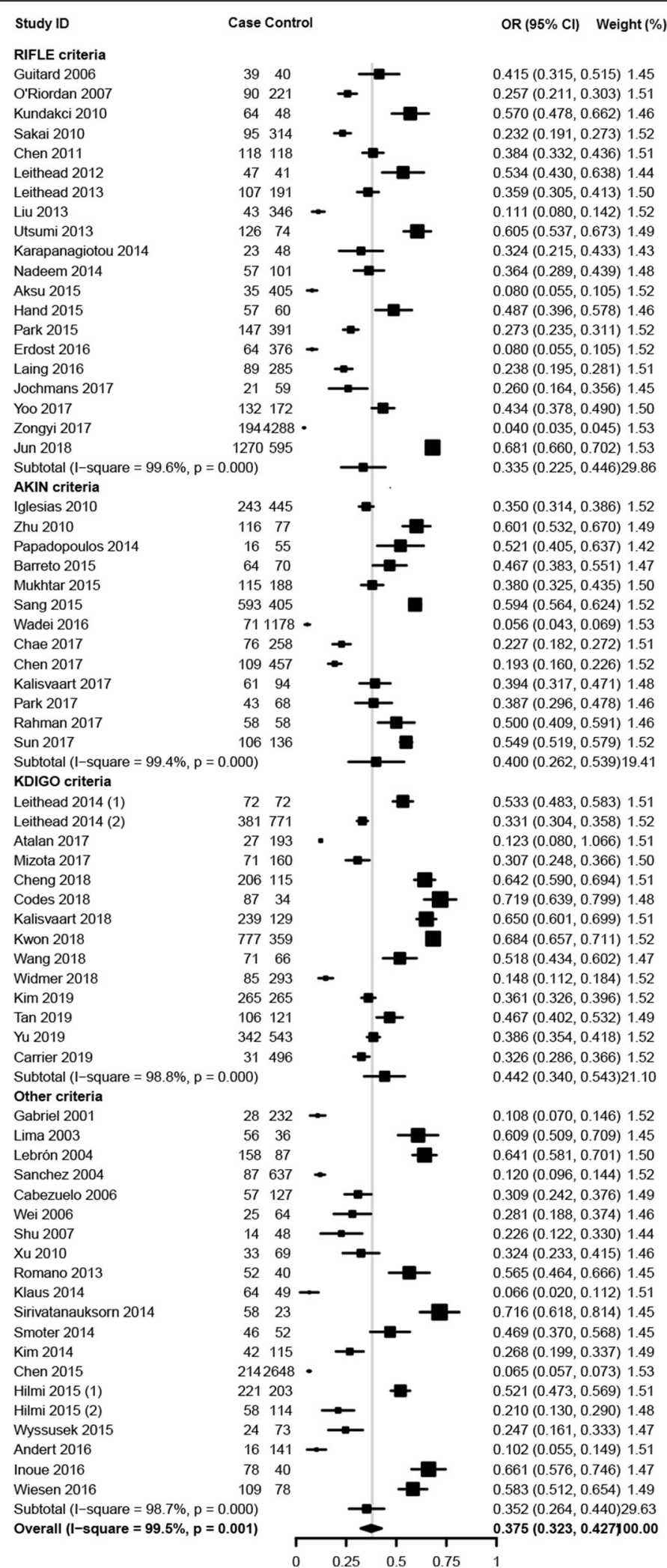

Fig. 2 Forest plots of the included studies assessing incidence rates of AKI after LT. A diamond data marker represents the overall rate from each included study (square data marker) and 95\% confidence interval 


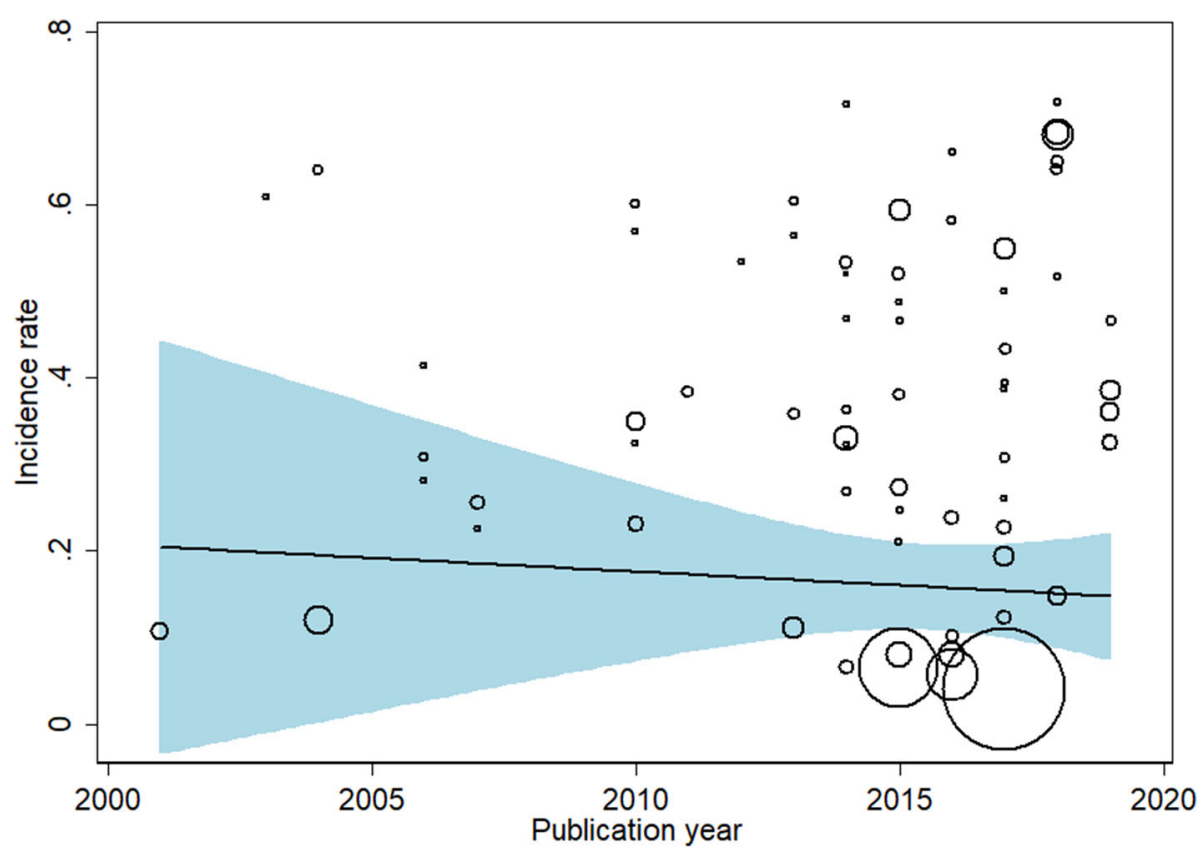

Fig. 3 Meta-regression of incidence rate of AKI after LT on publication year

piggyback surgical technique, split LT, venovenous bypass and intraoperative platelet transfusion.

\section{Postoperative factors}

Postoperative use of vasopressors $(\mathrm{OR}=2.234,95 \% \mathrm{CI}=$ $\left.1.431-3.488, \mathrm{I}^{2}=75.9 \%, P=0.000\right)$, overexposure to calcineurin inhibitor $(\mathrm{CNI})(\mathrm{OR}=2.762,95 \% \mathrm{CI}=1.737-$ $\left.4.391, \mathrm{I}^{2}=0.0 \%, \mathrm{P}=0.000\right)$, CNI without mycophenolate mofetil (MMF) $\left(\mathrm{OR}=2.087,95 \% \mathrm{CI}=1.404-3.103, \mathrm{I}^{2}=\right.$ $0.0 \%, \mathrm{P}=0.000)$, graft dysfunction $(\mathrm{OR}=3.124,95 \% \mathrm{CI}=$ $\left.2.036-4.795, \mathrm{I}^{2}=0.0 \%, \mathrm{P}=0.000\right)$, infection $(\mathrm{OR}=3.162$, $\left.95 \% \mathrm{CI}=2.315-4.320, \mathrm{I}^{2}=0.0 \%, \mathrm{P}=0.000\right)$ were associated with a higher risk for AKI after LT. Neither postoperative tacrolimus use nor postoperative hypoalbuminemia showed significant association with AKI after LT.

\section{Sensitivity analysis, subgroup analysis, meta-regression analysis, and publication bias}

Evident heterogeneity in the meta-analysis of 34 factors was found $\left(\mathrm{I}^{2}>50 \%\right.$ or $\left.P<0.1\right)$, and 28 factors could be further analyzed $(N \geq 3)$. The results of the sensitivity analysis are shown in Supplementary 7, Additional File. When 1 single study was excluded each time, the heterogeneity was obviously reduced $\left(\mathrm{I}^{2}\right.$ change $\left.>30 \%\right)$ for the following 10 modifiable factors: overweight, preoperative hypoalbuminemia, low GW/RBW, long CIT, long WIT, piggyback surgical technique, venovenous bypass, large intraoperative blood loss, intraoperative platelet transfusion, postoperative use of vasopressor. The source of heterogeneity may have been due to several design differences among the studies, including sample size, diagnostic criteria, duration of evaluation, cutoff point of factors, or insufficient adjustment for confounding factors. The details are shown in Supplementary 8, Additional File.

After conducting subgroup analysis by diagnostic criteria of AKI, the heterogeneity was obviously reduced $\left(\mathrm{I}^{2}\right.$ change $>30 \%$ ) for low GW/RBW, indicating diagnostic criteria might be the source of heterogeneity. The statistical significances were changed for preoperative hypoalbuminemia after conducting subgroup analysis. In the subgroup of RIFLE, preoperative hypoalbuminemia showed statistically significant association with AKI after LT without heterogeneity (see Supplementary 9, Additional File).

After conducting subgroup analysis by duration of evaluation, no obvious reduction of heterogeneity $\left(\mathrm{I}^{2}\right.$ change $>30 \%$ ) was observed for the aforementioned factors. However, the statistical significances were changed for several modifiable factors (see Supplementary 10, Additional File). In the subgroup of $\leq 7$ days, intraoperative platelet transfusion showed association with AKI after LT, whereas low GW/RBW showed no association with AKI after LT; in the subgroup of $>7$ days, major bleeding, large intraoperative RBC transfusion, postoperative use of vasopressor showed no association with AKI after LT.

After conducting subgroup analysis by statistical method, no obvious reduction of heterogeneity $\left(\mathrm{I}^{2}\right.$ change $>30 \%$ ) was observed for the aforementioned 
Table 1 Meta-analysis of risk factors for acute kidney injury after liver transplantation

\begin{tabular}{|c|c|c|c|c|c|c|c|}
\hline \multirow[t]{2}{*}{ Factor } & \multirow[t]{2}{*}{ type } & \multirow{2}{*}{$\begin{array}{l}\text { No. } \\
\text { of } \\
\text { study }\end{array}$} & \multirow[t]{2}{*}{ Sample } & \multirow{2}{*}{$\begin{array}{l}\text { Pooled OR }(95 \% \\
\text { Cl) }\end{array}$} & \multicolumn{2}{|c|}{ Heterogeneity } & \multirow[t]{2}{*}{$P$ value } \\
\hline & & & & & $1^{2}$ & $\mathrm{Chi}^{2}$ & \\
\hline \multicolumn{8}{|l|}{ Recipient factors } \\
\hline Age (per year) & C & 11 & 5476 & $1.006(0.998-1.015)$ & 78.3 & 0.000 & 0.155 \\
\hline Older age & B & 3 & 380 & $0.956(0.536-1.706)$ & 0.0 & 0.386 & 0.879 \\
\hline Female gender & B & 15 & 5399 & $1.479(1.186-1.845)$ & 42.5 & 0.042 & 0.001 \\
\hline Weight (per kg) & C & 2 & 288 & $1.021(0.996-1.046)$ & 0.0 & 0.875 & 0.098 \\
\hline BMI (per kg/m²) & C & 9 & 6558 & $1.080(1.062-1.099)$ & 0.0 & 0.904 & 0.000 \\
\hline Overweight & B & 5 & 2420 & $2.437(1.629-3.646)$ & 56.4 & 0.057 & 0.000 \\
\hline White race & $\mathrm{B}$ & 2 & 948 & $0.474(0.303-0.740)$ & 0.0 & 0.337 & 0.001 \\
\hline Hepatocellular carcinoma & B & 4 & 1027 & $0.681(0.316-1.469)$ & 66.6 & 0.030 & 0.328 \\
\hline Fulminant hepatic failure & B & 2 & 700 & $1.089(0.114-10.444)$ & 77.4 & 0.036 & 0.941 \\
\hline Alcoholic liver disease & B & 6 & 2320 & $1.747(1.326-2.302)$ & 0.3 & 0.414 & 0.000 \\
\hline Primary biliary cirrhosis & $\mathrm{B}$ & 2 & 466 & $0.657(0.276-1.566)$ & 32.3 & 0.224 & 0.343 \\
\hline Hepatitis B virus infection & B & 3 & 1367 & $0.710(0.486-1.039)$ & 18.7 & 0.292 & 0.078 \\
\hline Hepatitis $C$ virus infection & B & 5 & 2930 & $1.113(0.844-1.466)$ & 11.1 & 0.342 & 0.449 \\
\hline Cirrhosis & $\mathrm{B}$ & 2 & 887 & $2.171(1.322-3.566)$ & 0.0 & 0.518 & 0.002 \\
\hline Refractory ascites & B & 4 & 685 & $2.293(1.392-3.778)$ & 0.0 & 0.399 & 0.001 \\
\hline Pre-existing diabetes mellitus & B & 13 & 5763 & $1.390(1.199-1.611)$ & 0.0 & 0.480 & 0.000 \\
\hline Preoperative hypertension & B & 10 & 5544 & $1.291(0.814-2.045)$ & 64.7 & 0.003 & 0.278 \\
\hline Preoperative use of diuretic & B & 2 & 998 & $2.733(1.302-5.739)$ & 41.4 & 0.192 & 0.008 \\
\hline Child-Turcotte-Pugh grade $C$ & $\mathrm{~B}$ & 4 & 2031 & $1.876(1.205-2.922)$ & 76.7 & 0.005 & 0.005 \\
\hline Child-Turcotte-Pugh score (per score) & C & 5 & 2371 & $1.272(1.115-1.452)$ & 73.2 & 0.005 & 0.000 \\
\hline MELD (per score) & C & 23 & 10,444 & $1.035(1.024-1.045)$ & 38.7 & 0.031 & 0.000 \\
\hline High MELD score & B & 7 & 2174 & $1.986(1.474-2.676)$ & 8.7 & 0.363 & 0.000 \\
\hline Preoperative eGFR (per ml/min/1.73m²) & C & 2 & 456 & $1.007(1.000-1.015)$ & 0.0 & 0.785 & 0.050 \\
\hline APACHE II (per score) & C & 3 & 596 & $1.067(1.041-1.093)$ & 0.0 & 0.646 & 0.000 \\
\hline Preoperative serum creatinine (per $\mu \mathrm{mol} / \mathrm{L}$ ) & $\mathrm{C}$ & 3 & 582 & $0.998(0.952-1.046)$ & 88.6 & 0.000 & 0.931 \\
\hline Preoperative serum creatinine (per mg/dL) & C & 6 & 2422 & $2.337(1.215-4.497)$ & 81.0 & 0.000 & 0.011 \\
\hline High preoperative serum creatinine & B & 5 & 5498 & $2.155(1.219-3.811)$ & 67.2 & 0.016 & 0.008 \\
\hline Preoperative serum albumin (per g/dL) & C & 2 & 3001 & $0.539(0.460-0.632)$ & 0.0 & 0.740 & 0.000 \\
\hline Preoperative hypoalbuminemia & B & 3 & 958 & $1.127(0.259-4.905)$ & 96.1 & 0.000 & 0.874 \\
\hline Preoperative hemoglobin (per g/dL) & C & 4 & 4278 & $0.888(0.856-0.922)$ & 0.0 & 0.433 & 0.000 \\
\hline Preoperative anemia & B & 3 & 1410 & $1.621(1.073-2.449)$ & 24.5 & 0.266 & 0.022 \\
\hline \multicolumn{8}{|l|}{ Donor and graft factors } \\
\hline Cadaveric donor liver graft & B & 2 & 329 & $3.360(1.549-7.289)$ & 0.0 & 0.927 & 0.002 \\
\hline DCD organ & B & 3 & 1642 & $2.704(1.938-3.772)$ & 0.0 & 0.996 & 0.000 \\
\hline Donor age (per year) & C & 5 & 2170 & $1.004(0.991-1.017)$ & 49.5 & 0.095 & 0.578 \\
\hline Older donor age & B & 3 & 1470 & $1.213(0.799-1.840)$ & 23.6 & 0.270 & 0.364 \\
\hline Donor $\mathrm{BMl} \geq 30 \mathrm{~kg} / \mathrm{m}^{2}$ & B & 2 & 1309 & $2.672(1.173-6.085)$ & 57.8 & 0.124 & 0.019 \\
\hline Donor risk index (per point) & C & 2 & 1404 & $0.820(0.485-1.388)$ & 0.0 & 0.448 & 0.460 \\
\hline ABO-incompatible liver transplantation & B & 2 & 1274 & $2.761(1.602-4.759)$ & 0.0 & 0.751 & 0.000 \\
\hline Graft-recipient weight ratio & C & 3 & 3999 & $0.497(0.370-0.667)$ & 0.0 & 0.830 & 0.000 \\
\hline Low graft to recipient body weight ratio & B & 5 & 1565 & $1.902(1.013-3.568)$ & 52.3 & 0.078 & 0.045 \\
\hline Cold ischaemic time (per min) & C & 2 & 358 & $1.000(0.990-1.010)$ & 0.0 & 1.000 & 1.000 \\
\hline
\end{tabular}


Table 1 Meta-analysis of risk factors for acute kidney injury after liver transplantation (Continued)

\begin{tabular}{|c|c|c|c|c|c|c|c|}
\hline \multirow[t]{2}{*}{ Factor } & \multirow[t]{2}{*}{ type } & \multirow{2}{*}{$\begin{array}{l}\text { No. } \\
\text { of } \\
\text { study }\end{array}$} & \multirow[t]{2}{*}{ Sample } & \multirow{2}{*}{$\begin{array}{l}\text { Pooled OR (95\% } \\
\mathrm{Cl})\end{array}$} & \multicolumn{2}{|c|}{ Heterogeneity } & \multirow[t]{2}{*}{$P$ value } \\
\hline & & & & & $\mathrm{I}^{2}$ & $\mathrm{Chi}^{2}$ & \\
\hline Cold ischaemic time (per hour) & C & 7 & 2887 & $1.064(1.003-1.130)$ & 48.6 & 0.070 & 0.041 \\
\hline Long cold ischaemic time & B & 3 & 5220 & $1.408(0.907-2.187)$ & 73.1 & 0.024 & 0.128 \\
\hline Warm ischaemic time (per min) & C & 7 & 3427 & $1.018(1.007-1.029)$ & 53.6 & 0.044 & 0.001 \\
\hline Long warm ischaemic time & B & 3 & 5220 & $1.411(0.711-2.799)$ & 83.9 & 0.002 & 0.325 \\
\hline \multicolumn{8}{|l|}{ Surgical factors } \\
\hline Piggyback surgical technique & $\mathrm{B}$ & 3 & 903 & $0.556(0.195-1.585)$ & 81.8 & 0.004 & 0.272 \\
\hline Split liver transplantation & B & 2 & 1296 & $1.074(0.655-1.759)$ & 34.4 & 0.217 & 0.777 \\
\hline Venovenous bypass & $\mathrm{B}$ & 3 & 740 & $0.577(0.086-3.865)$ & 95.2 & 0.000 & 0.571 \\
\hline Intraoperative hypotension & B & 5 & 566 & $5.582(3.934-7.920)$ & 0.0 & 0.898 & 0.000 \\
\hline Intraoperative blood loss (per liter) & C & 5 & 1734 & $1.156(1.022-1.308)$ & 90.1 & 0.000 & 0.021 \\
\hline Large intraoperative blood loss & B & 6 & 5639 & $2.900(1.495-5.627)$ & 83.1 & 0.000 & 0.002 \\
\hline Intraoperative use of vasopressor & $\mathrm{B}$ & 13 & 4625 & $2.079(1.492-2.899)$ & 70.3 & 0.000 & 0.000 \\
\hline Intraoperative colloidal use & B & 3 & 495 & $2.447(1.508-3.973)$ & 0.0 & 0.630 & 0.000 \\
\hline Intraoperative RBC transfusion (per unit) & C & 15 & 8006 & $1.042(1.025-1.059)$ & 76.9 & 0.000 & 0.000 \\
\hline Intraoperative RBC transfusion (per liter) & C & 4 & 2253 & $1.196(1.143-1.253)$ & 11.4 & 0.336 & 0.000 \\
\hline Large intraoperative RBC transfusion & B & 11 & 3401 & $3.124(1.986-4.914)$ & 72.8 & 0.000 & 0.000 \\
\hline Intraoperative FFP transfusion (per unit) & C & 9 & 5202 & $1.027(1.021-1.032)$ & 3.8 & 0.403 & 0.000 \\
\hline Intraoperative platelet transfusion (per unit) & C & 3 & 1503 & $1.321(0.863-2.024)$ & 69.3 & 0.039 & 0.200 \\
\hline Intraoperative urine output (per mL) & C & 2 & 777 & $0.995(0.986-1.003)$ & 96.8 & 0.000 & 0.232 \\
\hline Postreperfusion syndrome & B & 9 & 4731 & $1.689(1.275-2.236)$ & 52.8 & 0.031 & 0.000 \\
\hline Duration of operation (per hour) & C & 4 & 1563 & $1.158(1.008-1.330)$ & 65.9 & 0.032 & 0.038 \\
\hline Long operation time & B & 2 & 738 & $1.485(0.937-2.353)$ & 0.0 & 0.470 & 0.093 \\
\hline \multicolumn{8}{|l|}{ Postoperative factors } \\
\hline Postoperative hypotension & B & 2 & 173 & $6.127(1.871-20.067)$ & 0.0 & 0.824 & 0.003 \\
\hline Large postoperative RBC transfusion & B & 2 & 308 & $5.051(2.387-10.691)$ & 0.0 & 0.899 & 0.000 \\
\hline Postoperative use of vasopressor & $\mathrm{B}$ & 4 & 4903 & $2.234(1.431-3.488)$ & 75.9 & 0.006 & 0.000 \\
\hline Postoperative peak AST (per u/L) & C & 2 & 232 & $3.687(1.081-12.575)$ & 79.7 & 0.026 & 0.037 \\
\hline Postoperative peak AST (per IU/L) & C & 2 & 196 & $1.451(0.618-3.410)$ & 84.2 & 0.012 & 0.393 \\
\hline Overexposure to $\mathrm{CNI}$ & B & 2 & 4682 & $2.762(1.737-4.391)$ & 0.0 & 0.857 & 0.000 \\
\hline No combined use of mycophenolate mofetil & B & 3 & 5220 & $2.087(1.404-3.103)$ & 0.0 & 0.899 & 0.000 \\
\hline Postoperative tacrolimus peak level (per ug/L) & C & 3 & 1675 & $0.983(0.951-1.017)$ & 50.0 & 0.135 & 0.326 \\
\hline Postoperative tacrolimus use & B & 4 & 2891 & $1.522(0.942-2.459)$ & 43.8 & 0.149 & 0.086 \\
\hline Postoperative hypoalbuminemia & $\mathrm{B}$ & 2 & 1528 & $0.718(0.261-1.976)$ & 95.6 & 0.000 & 0.522 \\
\hline Graft dysfunction & B & 3 & 1744 & $3.124(2.036-4.795)$ & 0.0 & 0.513 & 0.000 \\
\hline Infection & B & 8 & 1651 & $3.162(2.315-4.320)$ & 0.0 & 0.983 & 0.000 \\
\hline
\end{tabular}

OR odds ratio, $C$ continuous data, $B$ binary data, MELD model for end-stage liver disease, eGFR estimated glomerular filtration rate, APACHE acute physiology and chronic health evaluation, $D C D$ donation after cardiac death, $B M I$ body mass index, RBC red blood cell, FFP fresh frozen plasma, AST aspartate transaminase, CNI calcineurin inhibitor

factors, indicating statistical method was not the source of heterogeneity of aforementioned modifiable risk factors (see Supplementary 11, Additional File).

We also performed meta-regression analyses for factors with evident heterogeneity (see Supplementary 12, Additional File), and statistical method may partially explain the source of heterogeneity in intraoperative use of vasopressor. Based on the results from the funnel plot and Egger test (see Supplementary 13, Additional File), no evidence for publication bias was detected in factors described in $\geq 10$ studies. 


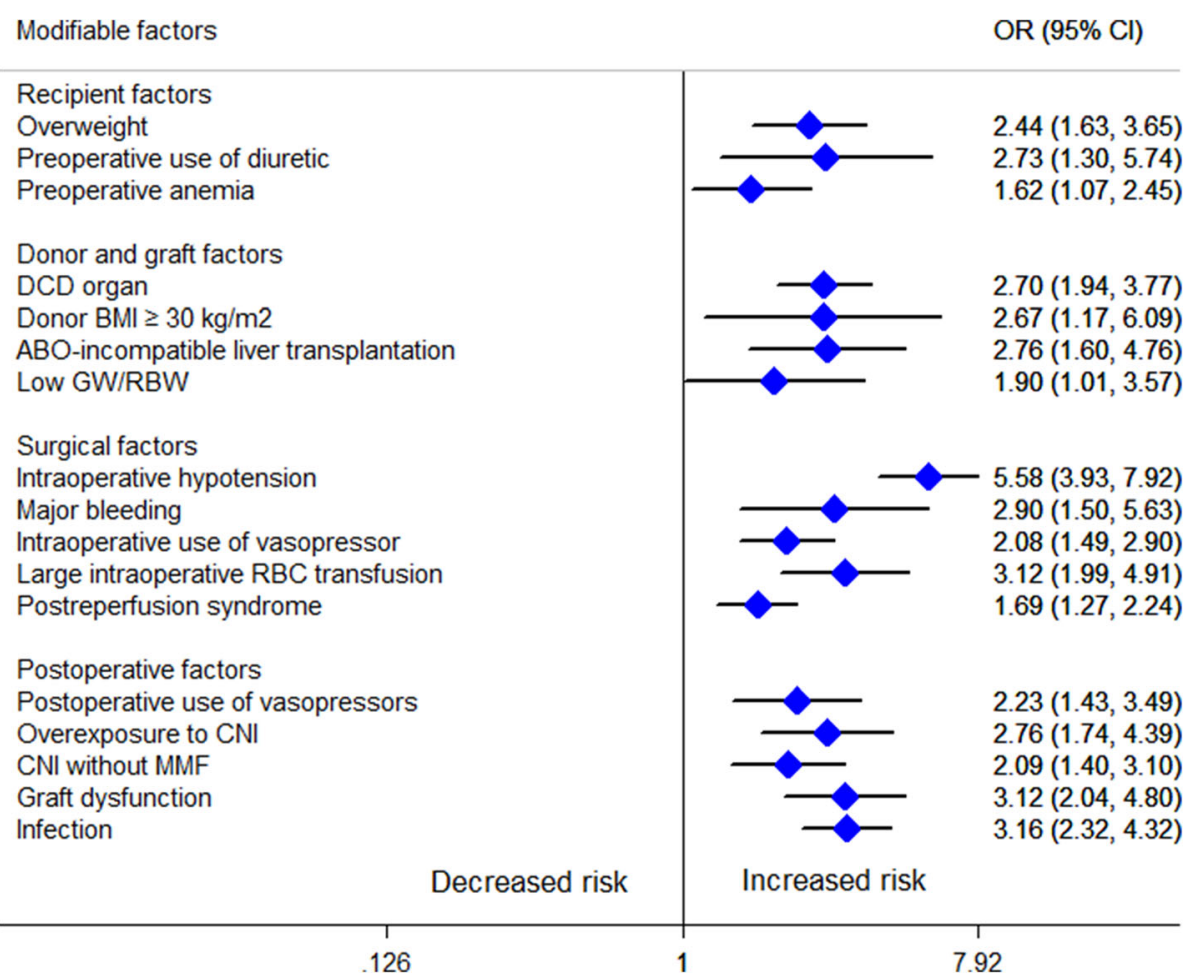

Fig. 4 Modifiable factors that show significant association with AKI after LT in the meta-analysis. OR, odds ratio; Cl, confidence interval; DCD, donation after cardiac death; BMI, body mass index; GW/RBW, graft weight to recipient body weight ratio; RBC, red blood cell; CNI: calcineurin inhibitor; MMF: mycophenolate mofetil

Modifiable factors

Recipient factors

Pulmonary hypertension

Preoperative hyponatraemia

Preoperative cerebrovascular diseases

Surgiacal factors

Intraoperative terlipressin therapy

Intraoperative use of diuretic (per mg)

Anhepatic time (per h)

Anesthetic time (per min)

Postoperative factors

Increased perioperative glucose variability

Postoperative use of aminoglycoside

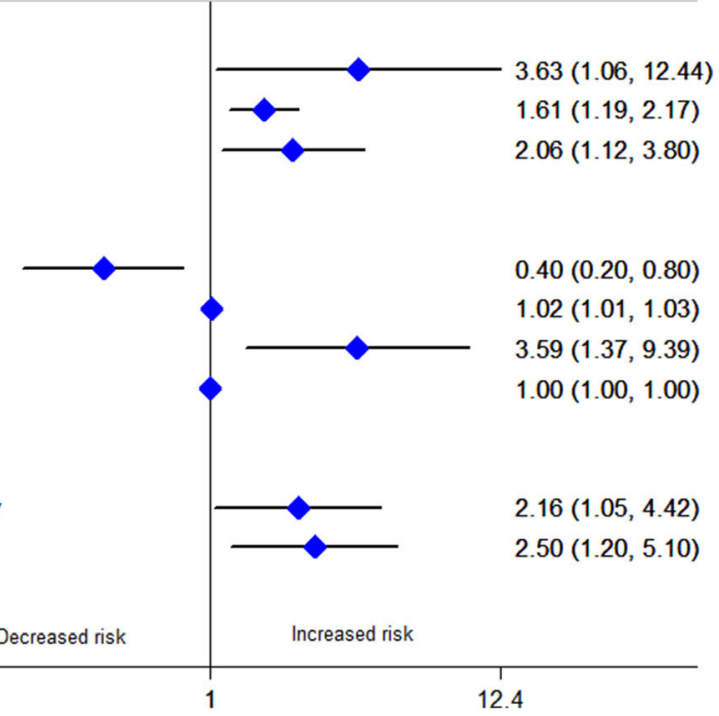

Fig. 5 Modifiable factors that show significant association with AKI after LT in the systematic review. OR, odds ratio; Cl, confidence interval 


\section{Systematic review}

A total of 75 factors were included only in the systematic review rather than the meta-analysis (see Supplementary 14, Additional File) because the assessment was performed in only 1 study. A total of 15 risk factors and 1 protective factor were associated with AKI after LT (here we only presented the factors adjusted for confounding factors with a relatively large population [> 300] to lower the error of estimates) (Fig. 5). In this systematic review, additional modifiable risk factors of AKI after LT were preoperative hyponatraemia, preoperative cerebrovascular diseases, pulmonary hypertension, increased perioperative glucose variability, long anesthetic time, intraoperative use of diuretic, long anhepatic time and postoperative aminoglycoside use. Besides, patients with intraoperative terlipressin therapy were associated with a decreased risk for AKI after LT.

\section{Discussion}

\section{Main findings}

In this systematic review and meta-analysis we have identified the modifiable risk factors of AKI after LT for the first time. Our work involved extensive analyses and shed new light on early identification and preventive strategies for AKI after LT. A total of 25 modifiable risk factors and 1 protective factor of AKI after LT were found (Fig. 6), of which 17 factors had data eligible for meta-analysis, including overweight, preoperative use of diuretics, preoperative anemia, $\mathrm{DCD}$ organ, donor $\mathrm{BMI} \geq$ $30 \mathrm{~kg} / \mathrm{m}^{2}$, ABO-incompatible LT, low GW/RBW, intraoperative hypotension, major bleeding, intraoperative use of vasopressor, large intraoperative RBC transfusion, postreperfusion syndrome, postoperative use of vasopressor, overexposure to $\mathrm{CNI}$, CNI without MMF, graft dysfunction and infection (Fig. 4).

\section{Explanation of results}

It is challenging to explore modifiable risk factors of AKI after LT because of numerous and heterogenous diagnostic criteria. Both criteria use Scr and urine output as markers of renal function [20]. Considering the retrospective nature of most observational studies, all included studies uniformly use Scr only to classify AKI after LT as detailed hourly urine output is often not available. The between-study heterogeneity may be the result of discrepancies in definition and classifications, various cutoff points of factors and different duration of evaluation. In addition, Scr can be influenced by dietary, volume overload, body muscle-mass and liver function [6]. In fact, Scr is a marker of renal function instead of kidney injury and can be delayed and insensitive under some circumstances [1]. As patients awaiting LT tend to have a reduced creatinine production compared with healthy subjects and fluid accumulation might mask the increase in Scr, it is likely that Scr overestimates the severity of preoperative renal function and delayed

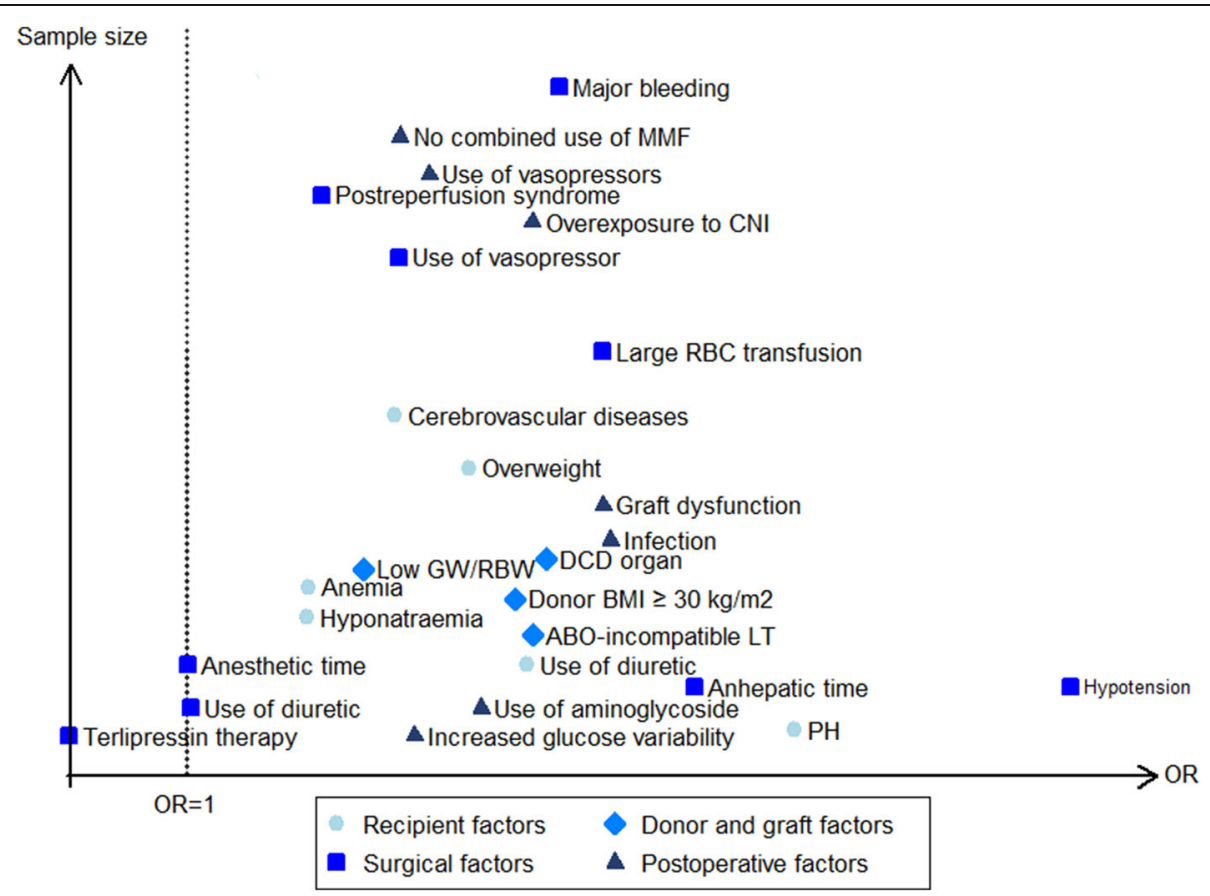

Fig. 6 Identified modifiable factors of AKI after LT. OR, odds ratio; DCD, donation after cardiac death; BMI, body mass index; GW/RBW, graft weight to recipient body weight ratio; RBC, red blood cell; CNI: calcineurin inhibitor; MMF: mycophenolate mofetil; LT, liver transplantation; PH, pulmonary hypertension 
diagnosis and underestimates the severity of postoperative AKI after LT. Another issue is the relevance of baseline Scr to the perioperative period. On the one hand, over-diagnosis can occur when the immediate Scr or the Scr after fluid resuscitation are selected as baseline Scr; on the other hand, comparing Scr after massive fluid administration in the postoperative period to preoperative baseline Scr can lead to under-diagnosis of AKI [21]. Thus, the diagnosis of AKI after LT faces challenges, and new biomarkers and adoption of standard definition are warranted.

That AKI after LT are multifarious in etiology is undeniable. As for recipient factors, overweight patient are at increased risk of AKI after LT, which is consistent with the findings of other clinical settings [22, 23]. Patients with high BMI are more likely to suffer from metabolic syndromes, including hypertension, dyslipidemia, cardiovascular and cerebrovascular diseases. Metabolic syndromes and obesity-related glomerulopathy may provide a vulnerable physiological reserve to handle the stress of hypoperfusion of kidney during surgery [24]. Though loop diuretic is widely used in different stages of AKI, data from randomized controlled trials and observational studies concerning the theoretical advantage of diuretics in preventing and treating AKI remain controversial and unproven [25-27]. Thus use of diuretics can lead to renal damage and nephrotoxicity and is recommended only if volume overload exists [28, 29]. Anemia may contribute to AKI through reducing the oxygen capacity of blood, enhancing oxidative stress and impairing hemostasis [30]. Therefore detecting and optimizing recipient preoperative hemoglobin status as early as possible is highly recommended in established consensus [31]. It is worth mentioning that sensitivity analysis revealed that the Cabezuelo (2006) study was the source of statistical heterogeneity in the meta-analysis for the association of preoperative hypoalbuminemia with AKI after LT. When this outlying study is removed, there was no evidence of heterogeneity in the remaining studies and the result showed that preoperative hypoalbuminemia increased the risk of AKI after LT $(\mathrm{OR}=2.134,95 \% \mathrm{CI}=$ $1.412-3.225, \mathrm{I}^{2}=0.0 \%, P=0.000$ ), which was broadly in line with previous studies cross different clinical settings [32]. The mechanism for the association of preoperative hypoalbuminemia with AKI after LT remains elusive, serum albumin may decrease the risk of AKI by maintaining renal perfusion, binding endogenous toxins and nephrotoxic drugs, alleviating oxidative damage, and delivering protective lysophosphatidic acid [32]. However, the association of serum albumin level and AKI might be Ushape [33], and there is evidence that administration of exogenous albumin failed to alter renal outcomes in the clinical scenario of living donor liver transplantation [34]. Hypotheses are proposed that not hypoalbuminemia itself but rather the underlying causes of it affect the occurrence of AKI after LT [6]. It appears that DCD organ is an independent risk factor for AKI after LT. Leithead hypothesized that hepatic ischaemia reperfusion injury (HIRI) is a leading cause for renal injury in recipients of DCD grafts [35]. It remains questionable whether the greater graft injury is attributed to the added donor WIT as previous studies have obtained conflicting results [36]. Unexpectedly, long WIT and long CIT are not risk factors for AKI after LT in our meta-analysis, though several previous studies have proved that they showed good performance in predicting AKI after LT. We postulate that though to some extent these risk factors reflect graft quality, they may be weak in promoting the occurrence of AKI after LT compared to other risk factors. Besides, with the improvement of organ procurement techniques, long ischemia time does not mean poor graft quality. Donor obesity and graft steatosis increase the susceptibility of the liver to HIRI, which is hypothesized as one of the driving forces of AKI after LT [35]. The exact mechanism for AKI in ABOincompatible LT patients has not been elucidated, probably owing to plasmapheresis, high isoagglutinin titer and enhanced immunosuppression [37, 38]. Low GW/RBW precipitates patients to persistent portal hypertension and a hyperdynamic state, which may impair the balance between vasodilatory and vasoconstrictor factors and lead to AKI after LT [39]. Overall these findings suggest that graft quality and HIRI may play an important role in the development of AKI after LT and optimizing graft quality and limiting HIRI is feasible measures to prevent AKI after LT.

With regard to surgical factors, our study indicates that intraoperative hypotension, major bleeding, use of vasopressor, large RBC transfusion, and postreperfusion syndrome (PRS) during operation are associated with increased risk of AKI after LT. Those variables reflect that hemodynamic instability, which exerts a major effect on reduction in renal blood flow and renal tissue hypoxia, may play a leading role in the development of AKI after LT. Notably, intraoperative hypotension had the highest OR as a modifiable risk factor for AKI after LT. Hypoperfusion and subsequent inflammation and neuroendocrine response to surgery are the frequent mechanisms affecting renal perfusion during perioperative period [2] Only when the mean arterial pressure is above the autoregulatory threshold can the kidney maintain glomerular filtration rate in the face of unstable arterial pressure and changing volume status. PRS leads not only to the release of cold and acidotic components by the graft but also pro-inflammatory cytokines that trigger inflammatory response and subsequent renal tubular injury [5]. In this regard, AKI after LT crosses the boundaries of traditional pathophysiological categories and encompasses pre-renal AKI and intrinsic renal AKI. Besides, RBC 
transfusion can induce oxidative stress and systemic inflammatory response syndrome, impair oxygen delivery and vascular regulation, release procoagulant phospholipids, and increase adhesiveness to vascular endothelium, thereby harming the kidneys [40]. In fact, many modifiable risk factors are interrelated and preventive strategies targeting multiple modifiable risk factors are crucially needed. Therefore, maintaining normovolaemia, reducing blood loss and avoiding unnecessary blood transfusion are of utmost importance for preventing the occurrence of AKI after LT. In addition, it is noteworthy that apart from norepinephrine, vasopressors like epinephrine, dopamine or phenylephrine did not show clinical benefit of renoprotective [41].

Concerning postoperative factors, patients with AKI after LT seemed to have more aggressive immunosuppressive regimen, more often had graft dysfunction, and experienced more hemodynamic insults immediately after operation. The relationship between graft dysfunction and AKI after LT is being increasingly recognized. As for graft dysfunction, evidences from animal experiments have shown that renal cells go through apoptosis during ischaemia reperfusion injury like hepatic cells after LT [42]. Intravascular oxidative stress and functional impairment of the mitochondria can trigger a systemic inflammatory response, thus the deleterious effects not only impair the liver, but also the kidney [43] In this regard, AKI after LT is not only a pathological condition of single organ failure but also can be seen as a marker of multi-organ injury [4], as there is increasing evidence that AKI directly contributes to remote organ injury and plays an active role in the progression of multi-organ dysfunction [2, 44]. Sepsis is the most frequent cause of AKI in the inpatient population and systemic inflammation resulting in tubular injury can account for sepsis-associated AKI irrespective of ischaemia as an initiating factor [45]. CNIs remain the mainstay of immunosuppression regimens after LT in spite of the well recognized nephrotoxicity. Renal artery vasoconstriction and development of thrombotic microangiopathy are presumed mechanism of CNI related injury [5]. Our work indicated that reduced or delayed CNI regimen combined with MMF decreased the incidence of AKI of LT. Overall tailoring the immunosuppression regimen in recipients at high-risk of developing severe AKI should be first priority in the immediate postoperative period.

The presence of heterogeneity was as expected, which may be due to the differences between individual studies, such as small sample size (postoperative use of vasopressor), different diagnostic criteria (intraoperative platelet transfusion), relatively long duration of evaluation (preoperative hypoalbuminemia, large intraoperative blood loss), relatively high cutoff point of definition (low GW/
RBW, long CIT) and insufficient adjustment for confounding factors. Therefore, we conducted specific analyses based on clinical and methodological characteristics of studies and adjusted for the heterogeneity as much as possible. Recruiting larger samples with unified diagnostic criteria and searching for appropriate statistical methods to adjust for confounding factors are necessary in future studies.

\section{Future directions}

Our study raised several questions that need to be addressed in future studies: (1) recipients with different baseline characteristics might show different sensitivity to perioperative precipitating factors and interventions. Therefore, risk stratification for AKI after LT using baseline characteristics is warranted in the future studies and clinical practice. (2) Considering that Scr can only serve as a retrospective marker of kidney function, it is reasonable to imagine that we might detect an ideal biomarker like highly sensitive troponin in the diagnosis of myocardial injury which is easily identifiable and will provide more timely and specific diagnosis of AKI in the near future. (3) At present, most risk prediction models for AKI after LT are lack of external validation in large multicentre cohorts, which significantly limits their clinical implementation. Therefore, finding reliable risk prediction tools (e.g. deep learning model [46] and automated clinical decision support systems [47]) using routinely measured variables continues to be intensive areas of research. (4) It is necessary to establish nonpharmacologic interventions specific for LT recipients based on the identified modifiable risk factors to improve prognosis of AKI after LT. To enable this, multidisciplinary cooperation including surgeons, anesthesiologists, and nephrologists are crucial in both clinical practice and future randomized controlled studies.

\section{Strengths and limitations}

There are several limitations in our study that should be addressed. First, only observational studies are included, thus some high-quality randomized controlled trial studies must have been missed, and our results cannot establish a cause-and-effect relationship. Second, studies included in this review represented a wide range of LT experience, institutional routines, and other possible existence of unknown or unmeasured factors that might influence the heterogeneity and potentially hamper the generalizability of the results. Third, different risk factors and predictors reported in various studies constrain us from accurately describing them in a similar manner. Fourth, not all studies made enough adjustment for confounding factors or provide precise effect size of multivariate analysis, and we can not fully unify the confounding factors due to the large number of studies. 
Fifth, inevitable heterogeneity may preclude conclusions regarding some factors that are likely to increase or decrease the development of AKI after LT. Finally, although efforts are made to eliminate duplicate data by title, author, cohort source, patient recruitment time, donor type and surgical technique, overlapping data may have been included in our study.

This is the first comprehensive systematic review and meta-analysis taking into account all modifiable risk factors of AKI after LT. The numerous modifiable risk factors could lay foundation for risk stratification, early identification, and effective prevention. Further highquality studies with larger sample sizes and randomized controlled trials targeting multimodal interventions are crucially needed.

\section{Conclusions}

This is by far the first study to quantitatively summarize the modifiable risk factors of AKI after LT. The modifiable risk factors identified in our study include overweight, preoperative use of diuretics, preoperative anemia, DCD organ, donor $\mathrm{BMI} \geq 30 \mathrm{~kg} / \mathrm{m}^{2}$, ABO-incompatible LT, low GW/ $\mathrm{RBW}$, intraoperative hypotension, major bleeding, intraoperative use of vasopressor, large intraoperative $\mathrm{RBC}$ transfusion, postreperfusion syndrome, postoperative use of vasopressor, overexposure to $\mathrm{CNI}$, CNI without MMF, graft dysfunction and infection. Effective interventions in the perioperative management and graft allocation and preservation may be promising to reduce the incidence of AKI after LT.

\footnotetext{
Abbreviations

AKI: acute kidney injury; Scr: serum creatinine; RIFLE: Risk, Injury, Failure, Loss of kidney function, End-stage renal failure; AKIN: Acute Kidney Injury Network; KDIGO: Kindney Disease: Improving Global Outcomes; LT: liver transplantation; RRT: renal replacement therapy; CKD: chronic kidney disease; ICU: intensive care unit; PRISMA: Preferred Reporting Items for Systematic Reviews and Meta-Analysis; MOOSE: Meta-analysis Of Observational Studies in Epidemiology; OR: odds ratio; Cl: confidence interval; NOS: NewcastleOttawa Scale; RBC: red blood cell; DCD: donation after cardiac death; BMl: body mass index; GW/RBW: graft weight to recipient body weight ratio; CIT: cold ischaemia time; WIT: warm ischaemia time; CNI: calcineurin inhibitor; MMF: mycophenolate mofetil; HIRI: hepatic ischaemia reperfusion injury; PRS: postreperfusion syndrome
}

\section{Supplementary Information}

The online version contains supplementary material available at https://doi. org/10.1186/s12882-021-02360-8.

\section{Additional file 1}

\section{Acknowledgments}

We acknowledge all staff who helped performed this study and particularly acknowledge Professor Mingxin Dong for the correction of grammar errors.

\section{Authors' contributions}

JZ conceived the idea, designed the study, performed statistical analyses and drafted the manuscript. XYZ and $L L$ conducted the literature research and evaluated the eligibility of all studies. XJM and GSM helped to data extraction and quality assessment. HCC contributed to conception, examination of the statistical methods and analyses, data interpretation, manuscript revision for critical intellectual content and supervision of the study. All authors read and approved the final manuscript.

\section{Funding}

The authors declare no funding.

\section{Availability of data and materials}

The datasets generated and/or analyzed during this study are included in this published article and its Supplementary information files.

\section{Declarations}

Ethics approval and consent to participate

Not applicable.

\section{Consent for publication}

Not applicable.

\section{Competing interests}

The authors declare that they have no competing interests.

\section{Author details}

1Department of Anesthesiology, The Affiliated Hospital of Qingdao University, School of Clinical Medicine, Qingdao University, No. 59, Haier Road, Qingdao 266100, Shandong Province, China. ${ }^{2}$ Department of Anesthesiology, West China Hospital, Sichuan University, Chengdu 610000, Sichuan Province, China.

Received: 2 November 2020 Accepted: 12 April 2021

Published online: 23 April 2021

\section{References}

1. Ronco C, Bellomo R, Kellum JA. Acute kidney injury. Lancet. 2019; 394(10212):1949-64. https://doi.org/10.1016/S0140-6736(19)32563-2.

2. Gumbert SD, Kork F, Jackson ML, Vanga N, Ghebremichael SJ, Wang CY, et al. Perioperative acute kidney injury. Anesthesiology. 2020;132(1):180-204. https://doi.org/10.1097/ALN.0000000000002968.

3. Susantitaphong P, Cruz DN, Cerda J, Abulfaraj M, Alqahtani F, Koulouridis I, et al. World incidence of AKl: a meta-analysis. Clin J Am Soc Nephrol. 2013; 8(9):1482-93. https://doi.org/10.2215/CJN.00710113.

4. Thongprayoon C, Kaewput W, Thamcharoen N, Bathini T, Watthanasuntorn K, Lertjitbanjong $P$, et al. Incidence and impact of acute kidney injury after liver transplantation: a meta-analysis. J Clin Med. 2019;8(3):372. https://doi. org/10.3390/jcm8030372

5. Durand F, Francoz C, Asrani SK, Khemichian S, Pham TA, Sung RS, et al. Acute kidney injury after liver transplantation. Transplantation. 2018;102(10): 1636-49.

6. de Haan JE, Hoorn EJ, de Geus HRH. Acute kidney injury after liver transplantation: recent insights and future perspectives. Best Pract Res Clin Gastroenterol. 2017;31(2):161-9. https://doi.org/10.1016/j.bpg.2017.03.004.

7. Kurzhagen JT, Dellepiane S, Cantaluppi V, Rabb H. AKI: an increasingly recognized risk factor for CKD development and progression. J Nephrol. 2020;33(6):1171-87. https://doi.org/10.1007/s40620-020-00793-2.

8. Walker $\mathrm{H}$, Bell S. Strategies to reduce perioperative nephrotoxicity. Semin Nephrol. 2019;39(5):442-53. https://doi.org/10.1016/j.semnephrol.2019.06. 004.

9. Mcinnes MDF, Moher D, Thombs BD, McGrath TA, Bossuyt PM, Clifford T, et al. Preferred Reporting Items for a Systematic Review and Meta-analysis of Diagnostic Test Accuracy Studies. Jama. 319(4):388.

10. Stroup DF, Berlin JA, Morton SC, Olkin I, Williamson GD, Rennie D, et al. Meta-analysis of observational studies in epidemiology: a proposal for reporting. J Am Med Assoc. 2000;283(15):2008-12. https://doi.org/10.1001/ja ma.283.15.2008.

11. Stang A. Critical evaluation of the Newcastle-Ottawa scale for the assessment of the quality of nonrandomized studies in meta-analyses. Eur J Epidemiol. 2010;25(9):603-5. https://doi.org/10.1007/s10654-010-9491-z.

12. Dersimonian R, Nan L. Meta-analysis in clinical trials. Control Clin Trials. 1986; 7(3):177-88. https://doi.org/10.1016/0197-2456(86)90046-2.

13. Dersimonian R, Laird N. Meta-Analysis in Clinical Trials Revisited. Contemp Clin Trials. 2015;45(Pt A):139-45. https://doi.org/10.1016/j.cct.2015.09.002. 
14. Higgins JPT, Thompson SG, Decks JJ, Altman DG. Measuring inconsistency in meta-analyses. Brit Med J. 2003;327(7414):557-60. https://doi.org/10.1136/ bmj.327.7414.557.

15. Cochrane handbook for systematic reviews of interventions, Version 5.1.0 (Online). [http://handbook-5-1.cochrane.org/]. Accessed 28 Apr 2020.

16. Easterbrook PJ, Gopalan R, Berlin JA, Matthews DR. Publication bias in clinical research. Lancet. 337(8746):867-72.

17. Duval S, Tweedie R. Trim and fill: a simple funnel-plot-based method of testing and adjusting for publication Bias in meta-analysis. Biometrics. 2000; 56(2):455-63. https://doi.org/10.1111/j.0006-341X.2000.00455.X.

18. Zongyi Y, Baifeng L, Funian Z, Hao L, Xin W. Risk factors of acute kidney injury after orthotopic liver transplantation in China. Sci Rep. 2017;7(1): 41555. https://doi.org/10.1038/srep41555.

19. Codes L, de Souza YG, D'Oliveira RAC, Bastos JLA, Bittencourt PL. Cumulative positive fluid balance is a risk factor for acute kidney injury and requirement for renal replacement therapy after liver transplantation. World J Transplant. 2018;8(2):44-51. https://doi.org/10.5500/wjt.v8.i2.44.

20. Thomas ME, Blaine C, Dawnay A, Devonald MA, Ftouh S, Laing C, et al. The definition of acute kidney injury and its use in practice. Kidney Int. 2015; 87(1):62-73. https://doi.org/10.1038/ki.2014.328.

21. Englberger L, Suri RM, Li Z, Casey ET, Daly RC, Dearani JA, et al. Clinica accuracy of RIFLE and acute kidney injury network (AKIN) criteria for acute kidney injury in patients undergoing cardiac surgery. Crit Care. 2011;15(1): R16. https://doi.org/10.1186/cc9960.

22. Suneja M, Kumar AB. Obesity and perioperative acute kidney injury: A focused review. J Critical Care. 2014;29(4):694 e691-694.e696.

23. Meersch M, Schmidt C, Zarbock A. Perioperative acute kidney injury. Anesth Analg. 2015;115(S2):1.

24. Kwakernaak AJ, Toering TJ, Navis G. Body mass index and body fat distribution as renal risk factors: a focus on the role of renal haemodynamics. Nephrol Dial Transplant 2013, 28 (Suppl 4):iv42-v49.

25. Ho KM, Power BM. Benefits and risks of furosemide in acute kidney injury. Anaesthesia. 2010;65(3):283-93. https://doi.org/10.1111/j.1365-2044.2009. 06228.x.

26. Libório $A B$, Barbosa ML, Sá VB, Leite $\Pi$. Impact of loop diuretics on critically ill patients with a positive fluid balance. Anaesthesia. 2020;75(S1). https://doi. org/10.1111/anae.14908.

27. Zhao GJ, Xu C, Ying JC, Lu WB, Hong GL, Li MF, et al. Association between furosemide administration and outcomes in critically ill patients with acute kidney injury. Crit Care. 2020;24(1):75. https://doi.org/10.1186/s13054-020-2 798-6.

28. Dreischulte T, Morales DR, Bell S, Guthrie B. Combined use of nonsteroidal anti-inflammatory drugs with diuretics and/or renin-angiotensin system inhibitors in the community increases the risk of acute kidney injury. Kidney Int. 2015;88(2):396-403. https://doi.org/10.1038/ki.2015.101.

29. Prieto-García L, Pericacho M, Sancho-Martínez SM, Sánchez Á, MartínezSalgado C, López-Novoa JM, López-Hernández FJ. Mechanisms of triple whammy acute kidney injury. Pharmacol Ther. 2016;167:132-45. https://doi. org/10.1016/j.pharmthera.2016.07.011.

30. Karkouti K, Wijeysundera DN, Yau TM, Callum JL, Cheng DC, Crowther M, et al. Acute kidney injury after cardiac surgery: focus on modifiable risk factors. Circulation. 2009;119(4):495-502. https://doi.org/10.1161/CIRCULA TIONAHA.108.786913.

31. Mueller MM, Van Remoortel H, Meybohm P, Aranko K, Aubron C, Burger R, et al. Patient blood management: recommendations from the 2018 Frankfurt consensus conference. Jama. 2019;321(10):983-97. https://doi. org/10.1001/jama.2019.0554.

32. Li N, Qiao H, Guo J-F, Yang H-Y, Li X-Y, Li S-L, Wang D-X, Yang L. Preoperative hypoalbuminemia was associated with acute kidney injury in high-risk patients following non-cardiac surgery: a retrospective cohort study. BMC Anesthesiol. 2019;19(1):171. https://doi.org/10.1186/s12871-0190842-3.

33. Wu P-H, Thongprayoon C, Cheungpasitporn W, Mao MA, Sakhuja A, Kashani K. U-shape association of serum albumin level and acute kidney injury risk in hospitalized patients. PLoS One. 2018;13(6):e0199153.

34. Mukhtar A, Masry AE, Moniem AA, Metini M, Fayez A, Khater YH. The impact of maintaining normal serum albumin level following living related liver transplantation: does serum albumin level affect the course? A pilot study. Transplant Proc. 2007;39(10):3214-8. https://doi.org/10.1016/j.transproceed.2 007.06.084.
35. Leithead JA, Rajoriya N, Gunson BK, Muiesan P, Ferguson JW. The evolving use of higher risk grafts is associated with an increased incidence of acute kidney injury after liver transplantation. J Hepatol. 2014;60(6):1180-6. https:// doi.org/10.1016/j.jhep.2014.02.019.

36. Leithead JA, Tariciotti L, Gunson B, Holt A, Isaac J, Mirza DF, et al. Donation After Cardiac Death Liver Transplant Recipients Have an Increased Frequency of Acute Kidney Injury. Am J Transpl. 12(4):965-75.

37. Jun I-G, Lee B, Kim S-O, Shin W-J, Bang J-Y, Song J-G, et al. Comparison of acute kidney injury between $\mathrm{ABO}$-compatible and $\mathrm{ABO}$-incompatible living donor liver transplantation: a propensity matching analysis. Liver Transpl. 2016;22(12):1656-65. https://doi.org/10.1002/lt.24634.

38. Yu JH, Kwon Y, Kim J, Yang S-M, Kim WH, Jung C-W, et al. Influence of transfusion on the risk of acute kidney injury: $\mathrm{ABO}$-compatible versus $\mathrm{ABO}$ incompatible liver transplantation. J Clin Med. 2019;8(11):1785. https://doi. org/10.3390/jcm8111785

39. Utsumi M, Umeda Y, Sadamori H, Nagasaka T, Takaki A, Matsuda H, et al. Risk factors for acute renal injury in living donor liver transplantation: evaluation of the RIFLE criteria. Transplant Int. 2013;26(8):842-52. https://doi. org/10.1111/tri.12138.

40. Tan L, Yang Y, Ma G, Zhu T, Yang J, Liu H, et al. Early acute kidney injury after liver transplantation in patients with normal preoperative renal function. Clin Res Hepatol Gastroenterol. 2019;43(4):475-82. https://doi.org/1 0.1016/j.clinre.2018.07.009.

41. Bellomo R, Wan L, May CN. Vasoactive drugs and acute kidney injury. Crit Care Med. 2008;36(10):2959. https://doi.org/10.1097/CCM.0b013e3181 $87 \mathrm{~b} 8 \mathrm{ac}$

42. Li B, Chen B, Zhang G, Wang K, Zhou L, Hu S. Cell apoptosis and fas gene expression in liver and renal tissues after ischemia-reperfusion injury in liver transplantation. Transplant Proc. 2010:42(5):1550-6. https://doi.org/10.1016/j. transproceed.2010.01.055.

43. Rahman S, Davidson BR, Mallett SV. Early acute kidney injury after liver transplantation: predisposing factors and clinical implications. World J Hepatol. 2017;9(18):823-32. https://doi.org/10.4254/wjh.v9.i18.823.

44. Gonsalez SR, Cortes AL, da Silva RC, Lowe J, Prieto MC. Acute kidney injury overview: from basic findings to new prevention and therapy strategies. Pharmacol Ther 2019.

45. Prowle JR, Bellomo R. Sepsis-associated acute kidney injury: macrohemodynamic and microhemodynamic alterations in the renal circulation. Semin Nephrol. 2015;35(1):64-74. https://doi.org/10.1016/j. semnephrol.2015.01.007.

46. Tomasev N, Glorot X, Rae JW, Zielinski M, Askham H, Saraiva A, et al. A clinically applicable approach to continuous prediction of future acute kidney injury. Nature. 2019;572(7767):116-9. https://doi.org/10.1038/s41586019-1390-1.

47. Kashani KB. Automated acute kidney injury alerts. Kidney Int. 2018;94(3):48490. https://doi.org/10.1016/j.kint.2018.02.014.

\section{Publisher's Note}

Springer Nature remains neutral with regard to jurisdictional claims in published maps and institutional affiliations.

\section{Ready to submit your research? Choose BMC and benefit from:}

- fast, convenient online submission

- thorough peer review by experienced researchers in your field

- rapid publication on acceptance

- support for research data, including large and complex data types

- gold Open Access which fosters wider collaboration and increased citations

- maximum visibility for your research: over $100 \mathrm{M}$ website views per year

At BMC, research is always in progress.

Learn more biomedcentral.com/submission 